\begin{tabular}{|c|l|}
\hline Title & $\begin{array}{l}\text { Speed-Power Performances of Quantum Wire Switches Controlled by Nanometer-Scale Schottky Wrap Gates for GaA s } \\
\text { based Hexagonal BDD Quantum LSIs }\end{array}$ \\
\hline Author(s) & Y umoto, Miki; Kasai, Seiya; Hasegawa, Hideki \\
\hline Citation & $\begin{array}{l}\text { Compound Semiconductors 2004 : Proceedings Of The Thirty-First International Symposium On Compound } \\
\text { Simiconductors Held In Seoul, Korea, 12-16 September 2004 (Institute of Physics Conference Series v.184), ISBN-10: } \\
\text { 0750310170, ISBN-13: 978-0750310178, pp. 213-216 }\end{array}$ \\
\hline Issue Date & 2005-06-15 \\
\hline Doc URL & http://hdl.handle.net/2115/30235 \\
\hline Rights & Copyright $\odot$ 2005 IOP Publishing Ltd. \\
\hline Type & bookchapter (author version) \\
\hline File Information & CS2004 184.pdf \\
\hline
\end{tabular}

Instructions for use 


\title{
Speed-Power Performances of Quantum Wire Switches Controlled by Nanometer-Scale Schottky Wrap Gates for GaAs based Hexagonal BDD Quantum LSIs
}

\author{
Miki Yumoto, Seiya Kasai and Hideki Hasegawa \\ Research Center for Integrated Quantum Electronics and Graduate School \\ of Information Science and Technology, Hokkaido University, N14, W9, \\ Sapporo, 060-0814, Japan
}

\begin{abstract}
This paper investigates the basic speed-power performance of Schottky wrap gate (WPG) controlled GaAs quantum wire (QWR) switches for hexagonal BDD quantum circuits. The estimated power-delay products (PDPs) of the switches were less than $10^{-20} \mathrm{Js}$ at low temperatures, being smaller than those of the latest Si CMOSFETs. Direct microwave measurements and equivalent circuit analysis confirmed capability of $\mathrm{GHz}$ clock operation.
\end{abstract}

\section{Introduction}

Nanometer-scale quantum devices will play important roles in the advanced information technology (IT) of the future ubiquitous network society. This is because of their small sizes and of smallest power-delay product (PDP) values near the quantum limit, both of which may lead to high-density, high-speed and ultra low-power quantum (Q-) LSIs beyond the scaling limit of the Si CMOS technology. However, quantum devices show weak current drivability and poor threshold control, making them unsuitable to the conventional logic gate architecture. To overcome this difficulty, we have recently proposed a novel hexagonal binary decision diagram (BDD) quantum logic circuit approach [1], and demonstrated small scale circuits up to 2-bit quantum adders [2].

This paper investigates the basic speed power performance of Schottky wrap gate (WPG) controlled GaAs quantum wire (QWR) switches for hexagonal BDD Q-LSIs.

\section{Basic Concept and Device Structure}

\subsection{Hexagonal BDD quantum logic circuit approach}

As shown in Fig. 1(a), the hexagonal BDD quantum logic circuit consists of node devices and root and terminal electrodes formed on a hexagonal closely packed planar QWR network. Each node device has one entry- and two exit-branches as shown in Fig. 1(b), and selects the exit path for the incoming information messenger according to the gate input. The value of a logic function is determined whether the information messenger coming from the root terminal reaches terminal 1 or terminal 0 after traveling through an array of node devices. Any combinational logic function can be realized as a hexagonal BDD graph without nanowire crossover. If the messenger is a single or a few electrons, an ultra small PDP near the quantum limit can be realized. 


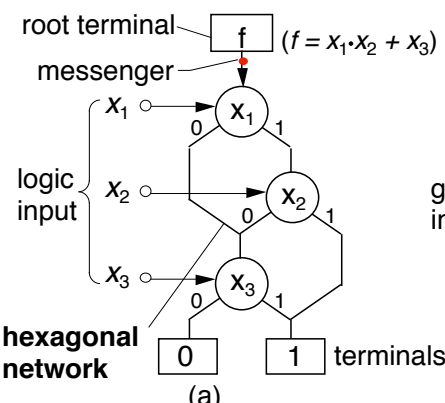

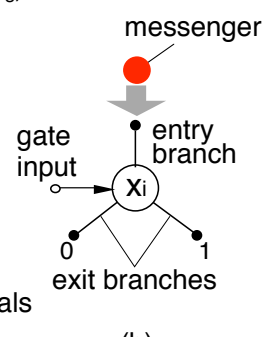

(b)

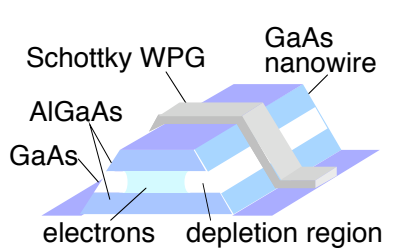

(c)

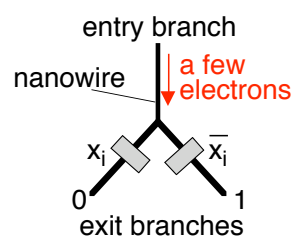

(d)

Figure 1. (a) Hexagonal BDD circuit, (b) function of node device, (c) WPGcontrolled QWR switch and (d) node device used in this study.

\subsection{Device structure and fabrication process}

In this study, we realized QWR switches by controlling an etched AlGaAs/GaAs nanowire with a nanometer-scale Schottky wrap gate (WPG) [3] as shown in Fig. 1(c). The gate has a simple structure suitable for planar integration and can provide tight potential control. A hexagonal BDD node device can be constructed by a pair of WPG QWR switches, as shown in Fig. 1(d). Here path switching between zero-th and first steps of quantized conductance is made by applying gate voltages to WPGs on two exitbranches in a complementary fashion. For device fabrication, Y-branch structures were formed on an $\mathrm{AlGaAs} / \mathrm{GaAs}$ heterostructure wafer by electron beam lithography and wet chemical etching. After ohmic contact formation for terminals, nanometer-scale Al Schottky WPGs were formed by EB lithography and lift-off process.

\section{Experimental Results and Discussion}

\subsection{Quantum transport and path switching characteristics}

WPG QWR switches showed excellent gate control characteristics from low temperatures up to room temperature. They exhibited clear conductance quantization at low temperatures up to $70 \mathrm{~K}$, as shown in Fig. 2 for a QWR switch with the wire width, $W$, of $580 \mathrm{~nm}$ and the gate length, $L_{\mathrm{G}}$, of $400 \mathrm{~nm}$. BDD node devices having them exhibited clear path switching operation between zero-th and first quantized conductance steps in quantum regime as shown in Fig. 3. At room temperature, they also showed clear path switching operation. This is because each switch operates as a classical high electron mobility transistor (HEMT) switch, controlling flow of many electrons.

\subsection{Power-delay product}

The value of PDP can be estimated roughly by $C_{\mathrm{G}} \Delta V_{\mathrm{G}}{ }^{2}$, where $C_{\mathrm{G}}$ is the gate capacitance and $\Delta V_{\mathrm{G}}$ is the gate voltage swing for switching. Obviously, the voltage slope of the first conductance step, $\gamma=\mathrm{d} G / \mathrm{d} V_{\mathrm{G}}$, plays an important role in determining $\Delta V_{\mathrm{G}}$. The measured

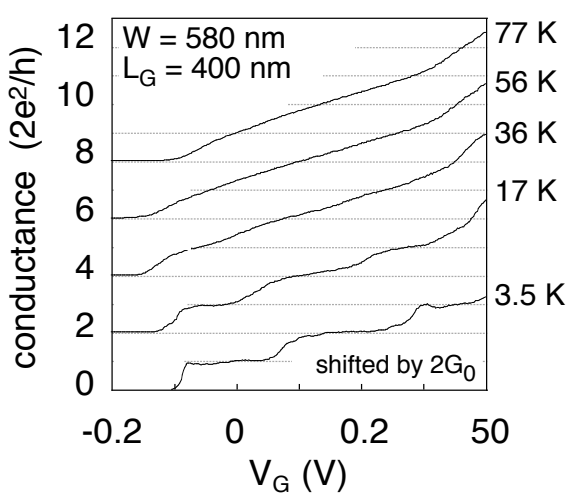

Figure 2. Conductance quantization.

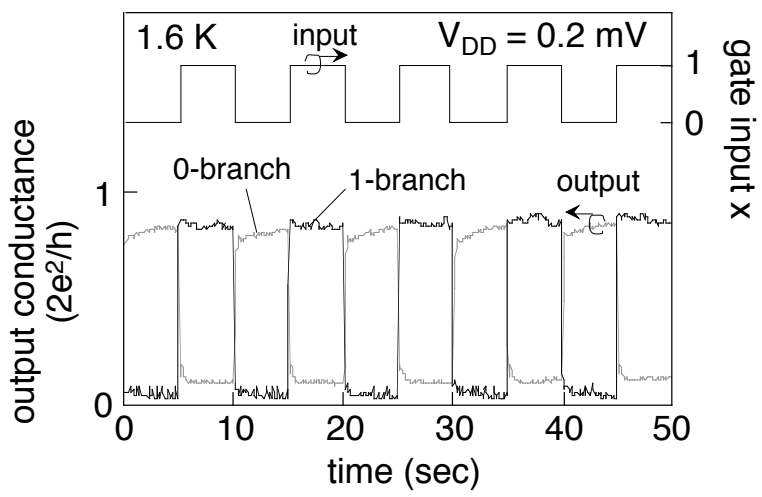

Figure 3. Path switching. 


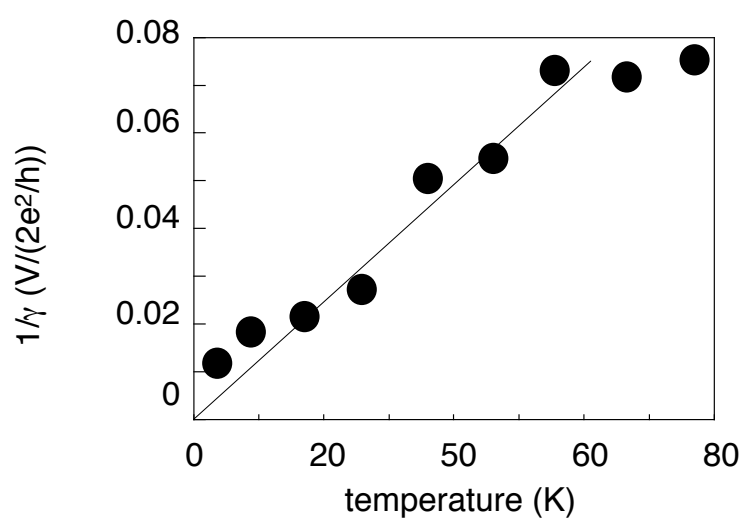

Figure 4. $1 / \gamma$ vs. $T$.

values of $1 / \gamma$ from Fig. 2 are plotted as a function of temperature in Fig. 4. A linear relation was obtained up to $60 \mathrm{~K}$ in agreement with a theory [4] which predicts

$$
\gamma=\frac{G_{0}}{4 k_{\mathrm{B}} T} \frac{d \mu}{d V_{\mathrm{G}}}
$$

where $\mu$ is a chemical potential in the QWR. From the plot, $\mathrm{d} \mu / \mathrm{d} V_{\mathrm{G}}=0.27$ was obtained. This value is about 10 times larger than that of the standard split gate structure [5]. Thus, the WPG can switch with 100 times smaller power consumption.

The value of the gate capacitance $C_{\mathrm{G}}$ of the QWR switch can be estimated by

$$
C_{\mathrm{G}}=\frac{q \Delta n_{1 \mathrm{D}} \cdot L_{\mathrm{G}}}{\Delta V_{\mathrm{G}}}
$$

where $n_{1 \mathrm{D}}$ is the electron one-dimensional density in the QWR switch. Its value was evaluated from gate-dependent Shubnikov-de-Haas $(\mathrm{SdH})$ oscillation measurement [6]. As a result, $C_{\mathrm{G}}=0.6 \mathrm{fF}$ and $C_{\mathrm{G}}=13 \mathrm{fF}$ were obtained for switches with $L_{\mathrm{G}}=65 \mathrm{~nm}$ and $L_{\mathrm{G}}=630 \mathrm{~nm}$, respectively. The corresponding values of PDP at $1.6 \mathrm{~K}$ were found to be $10^{-21} \mathrm{~J}$ and $10^{-20} \mathrm{~J}$, respectively. As expected, a smaller PDP value was realized by reducing $L_{\mathrm{G}}$. These PDP values are three or four orders of magnitude smaller than that of the latest $\mathrm{Si}$ MOSFET with $L_{\mathrm{G}}=20 \mathrm{~nm}$ at room temperature [7].

\section{2. $\quad$ Switching speed of WPG QWR switches}

Direct switching speed measurements of a single WPG QWR at low temperatures could be done only up to $10 \mathrm{MHz}$ due to instrumental limitation and high-impedance nature of the device. Up $10 \mathrm{MHz}$, fabricated devices worked correctly without problems.

However, an estimate of the switching speed by $C_{\mathrm{G}} / G_{0}$ indicates feasibility of $\mathrm{GHz}$

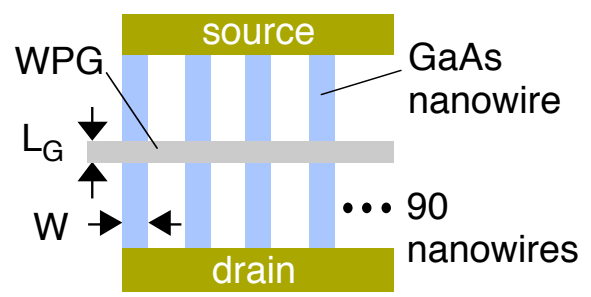

(a)

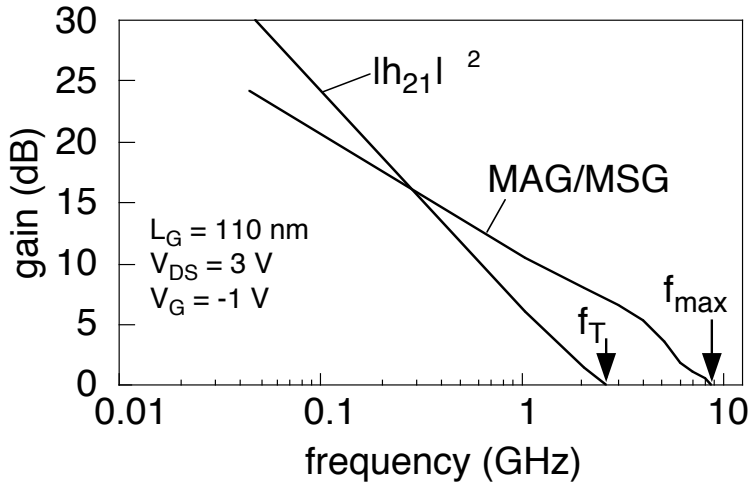

(b)

Figure 5. (a) Structure of device for RF measurements and (b) gain plots. 
switching. To confirm this, special devices having 90 nanowires in parallel as shown in Fig. 5(a) were fabricated, since quantum devices are high impedance devices and do not fit to $50 \Omega$-measurement systems. Microwave measurements were then performed directly using an Agilent vector network analyzer 8510C. This could only be done at room temperature due to difficulty of RF-probing at low temperatures. Figure 5(b) shows the measured current gain, $\left|h_{21}\right|^{2}$, and MAG/MSG of the device with $L_{\mathrm{G}}=110 \mathrm{~nm}$, giving a cut-off frequency, $f_{\mathrm{T}}$, of $2.5 \mathrm{GHz}$ and the maximum oscillation frequency, $f_{\max }$, of $9 \mathrm{GHz}$. The device with $L_{\mathrm{G}}=630 \mathrm{~nm}$ gave $f_{\mathrm{T}}=1 \mathrm{GHz}$ and $f_{\max }=5 \mathrm{GHz}$. The results directly confirms feasibility of GHz clock operation of the hexagonal BDD circuits. They also indicate that switching speed becomes faster as the gate length is reduced.

Evaluation of the device parameters were made by using a standard equivalent circuit model for a HEMT shown in Fig. 6(a). The parameter values extracted by fitting the model to measured S-parameters are shown for a device with $L_{\mathrm{G}}=65 \mathrm{~nm}$ on the right of Fig. 6(a). The value of gate-source capacitance, $C_{\mathrm{gs}}$, of a single WPG QWR was 0.33 $\mathrm{fF}$ in reasonably good agreement with the above value by $\mathrm{SdH}$ measurements. The value of $R_{\mathrm{i}}$ was found to be in good agreement with the expected value of gate-to-source series resistance of the wire. It was also found necessary to include parasitic capacitances, $C_{1}$ and $C_{2}$, to obtain good fitting, as shown in Fig. 6(b). We tentatively assume that these parasitic capacitances are due to surface states. Thus, faster switching speeds can be realized by reducing WPG sizes and providing good surface passivation.

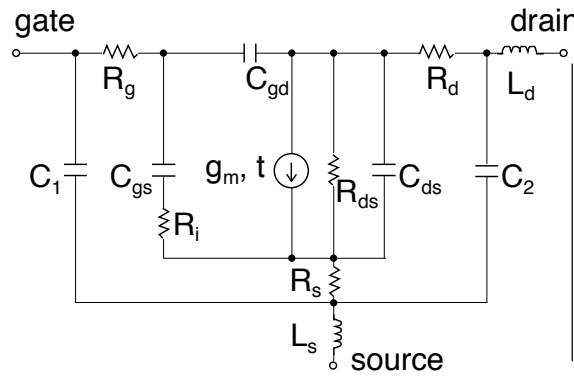

(a)

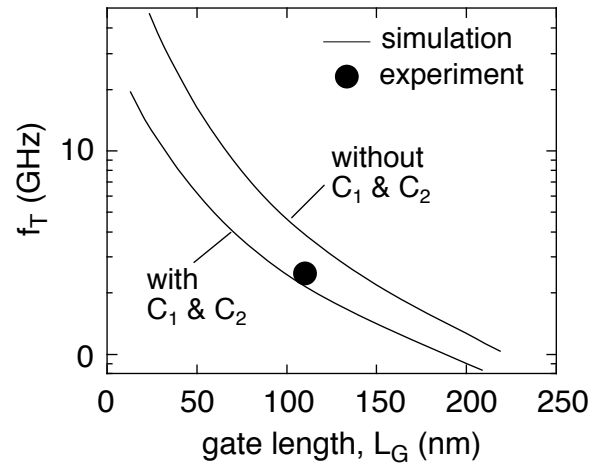

(b)

Figure 6. (a) HEMT model for GaAs QWR switch and (b) $f_{\mathrm{T}}$ vs. $L_{\mathrm{G}}$ for two cases.

\section{Conclusion}

PDP values of the WPG controlled GaAs QWR switches for hexagonal BDD quantum circuits are less than $10^{-20} \mathrm{Js}$ at $1.6 \mathrm{~K}$, being much superior to latest Si CMOSFETs. Direct microwave measurements confirmed feasibility of $\mathrm{GHz}$ clock operation.

The work reported here is supported in part by a 21C COE Project at Hokkaido University on "Meme-Media Technology Approach to the R\&D of Next-Generation Information Technologies" from MEXT, Japan.

\section{References}

[1] Kasai S and Hasegawa H 2002 IEEE Electron Device Lett. 23446

[2] Kasai S, Yumoto M and Hasegawa H 2003 Solid State Electronics 47 199-204

[3] Kasai S 1997 Jpn. J. Appl. Phys. 361678

[4] Bagwell P F and Orlando T P 1989 Phys. Rev. B 401456

[5] Wees B J van 1991 Phys. Rev. B 4312431

[6] Yumoto M, Kasai S and Hasegawa H 2002 Appl. Surf. Sci. 190 242-246

[7] Chau R 2001 Nanoelectronics Workshop (Kyoto) 